\title{
SIMULATION OF LASER TRIANGULATION SENSORS SCANNING FOR DESIGN AND EVALUATION PURPOSES
}

\author{
Cajal, C. ${ }^{*}$; Santolaria, J.**; Samper, D. ${ }^{* *} \&$ Garrido, A.** \\ ${ }^{*}$ Centro Universitario de la Defensa Zaragoza, University of Zaragoza, Spain \\ ** Department of Design and Manufacturing Engineering, University of Zaragoza, Spain \\ ${ }^{* * *}$ Department of Marketing Management and Market Research, University of Zaragoza, Spain \\ E-Mail: ccajal@unizar.es
}

\begin{abstract}
Nowadays, computerized dimensional inspection is included in most manufacturing processes. Industrial vision systems, laser triangulation sensors (LTS) in particular, are responsible for ensuring dimensional and geometrical tolerance compliance. Computer-aided design / computer-aided engineering $(\mathrm{CAD} / \mathrm{CAE})$ plays an important role in the manufacturing process. However, in the design and engineering of LTS, there is no equivalent computer-aided tool to provide assistance to the process definition. This paper presents a simulation environment to assist with this issue. Based on the CAD files of the parts, all the parameters of an LTS sensor and its scanning strategy can be simulated in a virtual Direct3D environment. This simulation provides a re-creation of the camera acquisition, allowing collision and occlusion detection and ensures complete and effective digitalization. Furthermore, the capability of the process of tolerance compliance evaluation can be verified. Once the effectiveness of the process is proven, the simulation can be employed for continuous performance improvement and the incorporation of new parts.

(Received in May 2014, accepted in December 2014. This paper was with the authors 1 month for 1 revision.)
\end{abstract}

Key Words: Sensors, Scanning, Digitalization, Industrial vision, Simulation

\section{INTRODUCTION}

Quality inspection control systems are crucial to present-day production systems. It is not possible to conceive of any production process without the implementation of the corresponding control. Currently, controls are not limited to final product quality assurance; instead, any single step in the manufacturing of a product can be subject to evaluation. The time and cost invested in quality improvement activities through the production line are amplified back [1]. There are plenty of aspects in which the quality of a manufacturing process can be quantified. In industry, these aspects can be classified into dimensional quality, structural quality, surface quality and operational quality [2]. Widespread dimensional quality assurance requires $100 \%$ verification and therefore it is essential to put in place nondestructive verification mechanisms. These mechanisms consist of $3 \mathrm{D}$ data acquisition techniques that are processed in real time to decide whether the quality remains within specified boundaries.

Data acquisition techniques are commonly classified into contact and non-contact techniques [3]. Contact or tactile devices are those that touch the surface of the object with a mechanical sensor, such as Coordinate Measuring Machines (CMM) or an Articulated Arm CMM (AACMM) that uses a mechanical probe. On the other hand, non-contact devices are based on magnetic, optical or acoustic principles to obtain 3D data from an object. Returning to dimensional quality assurance, contact measurement mechanisms are slow, since they need to ensure physical contact with a part surface for every point that is sampled [4]. For this reason, there is the risk of the probing force, that can deform a part, either disabling the measurement or even damaging the surface if the deformation is permanent [5]. However, 
non-contact measurement systems are up to 100 times faster [4] and, as the name indicates, they are harmless to the part.

Among non-contact systems, some of which are very affordable [6], industrial vision laser triangulation systems (LTS) are the most commonly used due to the possibility of the simultaneous acquisition of a points profile, thus multiplying the speed of 3D objects measurement. Basic laser triangulation sensors are composed of a laser projecting device and a digital camera. The camera captures images of the diffuse reflection of a laser on the surface to be measured. Using the triangulation principle, the systems are able to provide the coordinates from the laser line image pixels, the calibration of the camera and the predetermined laser plane equations [7].

Typically, an industrial inspection system computes information from raw images according to the following sequence of steps [2]: image acquisition, image processing, feature extraction and decision-making. The image acquisition depends on various factors. Some of them could be considered constructive and the rest are considered to be operative ones. The selection of components, degrees of freedom (DoFs) and the physical design of the support structure are fixed, while the frame rate, trajectories, speed and region of interest (ROI) can be parameterized in real time [2].

The first design criterion is the image acquiring device selection. Without going into specifics, the market offer of industrial purpose cameras is huge. Optics selection expands the possibilities still more. Each device has its own features, the most relevant being resolution, maximum frame rate, sensor noise and external trigger capabilities [2]. Optics selection defines the focal distance and, in combination with the camera, the field of view (FOV).

A laser scanning operation consists of a relative movement of the sensor (camera and laser) with respect to the part to be digitalized. Therefore, these movements depend on the machine that performs the movement and on its degrees of freedom. Using an LTS, a CMM can perform at least 3 independent lineal movements [7], while robots usually have 6 angular degrees of freedom. An additional movement axis can be established if required [8]. The paths required to obtain the measurement of any feature within the part can be complex, and this problem is addressed in many investigations [9-11]. Motion planning of laser sensors benefits from its similarity to production technologies where path-oriented systems are widely used [12], including milling, grinding, roller burnishing [13] and spraying. In addition to concern with tool collision, with a laser sensor there are also concerns with occlusion of either the laser or the camera $[11,14,15]$.

In addition to the design parameters that have been mentioned, certain other parameters have to be adjusted depending on the requirements of the measurement. These parameters, known as operation parameters, are easily modified and can be adjusted during scanning. Taking into account the constraints of the selected degrees of freedom, the scanning trajectory is one of these parameters. Speed and acceleration are normally subject to variation during the scanning [16], as are the region of interest (ROI) and frame rate of the camera.

Having dealt with ideal parameters, the real parameters need to be taken into consideration. The actual measurement is affected, as well, by other undesired effects like random errors caused by speckles in the laser images due to cancellation and reinforcement of the light wave amplitude [9]. In this respect, Smith and Zheng [17] presented a modelling technique to simulate the effect of different factors, including incident angle and surface reflectivity, on accuracy. In addition, optical distortion significantly affects the results [1820], and is relevant for short focal distance optics. Thus, all these effects are considered as a source of uncertainty [21].

It has to be kept in mind that the LTS is presented as a quality assurance method and therefore its performance is the final goal. Basically, for measurement systems, unwise strategy planning can be the largest uncertainty contributor [22]. Besides, parts verification 
can be a significant cost, so a proper sampling strategy becomes a must. Sampling of the part can be classified as blind, results-dependent and process-dependent [23], based on the quality requirements of the process. Each of them has to supply enough information and guarantee that there are no potential movement overlaps.

In this paper, we present a simulation environment where design and operative parameters of LTS quality controls can be managed. Furthermore, the actual scanning conditions are reproduced to address measurement challenges in advance and therefore reduce cost and ensure viability.

\section{PROBLEM UNDER STUDY}

The increasing complexity of the parts designed in industry makes the design of vision systems even more difficult. There is a tendency for the market to provide flexible systems that can meet many different requirements. In some cases, basic image processing software is sufficient. However, in specific tolerance validation by industrial vision systems, the image acquisition step is more complex. Most of the cases require specific tools to be manufactured, which implies a long time and increases the final cost. Validation and forecasting of the measurement process are the problems addressed in this paper. Fig. 1 presents a block diagram of the proposed simulation environment.

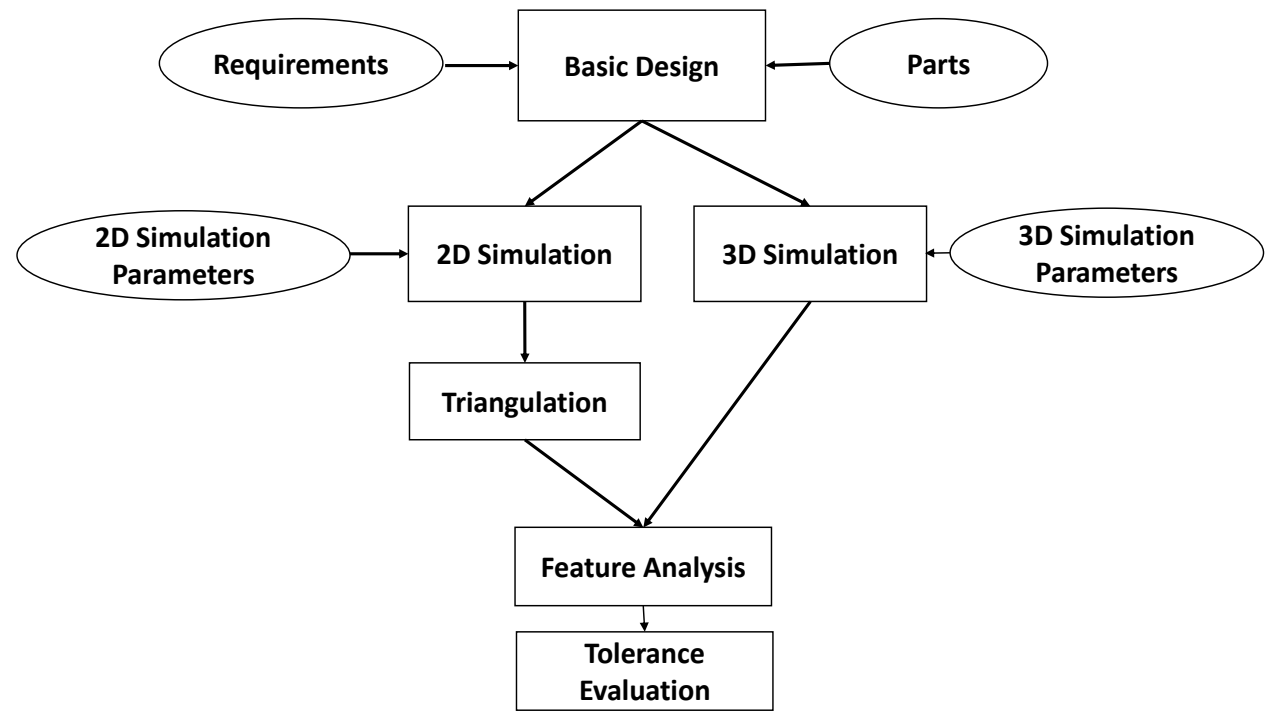

Figure 1: LST simulation environment block diagram.

First of all, the system needs to be modelled, each component with its own geometric parameters:

- Camera: various camera operation parameters, position and spatial disposition and noise of the sensor.

- Optic: focal length, aperture.

- Laser: disposition, focus.

- Part: CAD, dimensional range of each of the features.

- Degrees of freedom: movement capabilities of each component or an ensemble of some of them.

- Scanning: speed, acceleration and trajectories.

- Output: the data collected should be compatible with the processing data. It could be either images or a cloud of points if the 2D processing is already done. In Fig. 2 a the output is superposed on the CAD. Fig. $2 \mathrm{~b}$ shows the simulation where the output is calculated. 


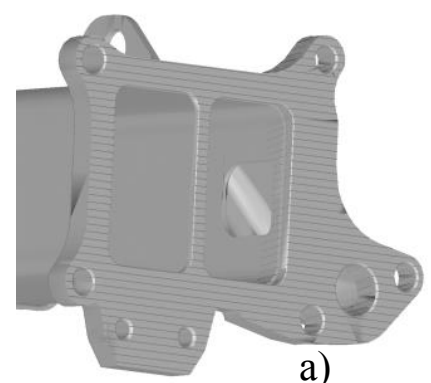

a)

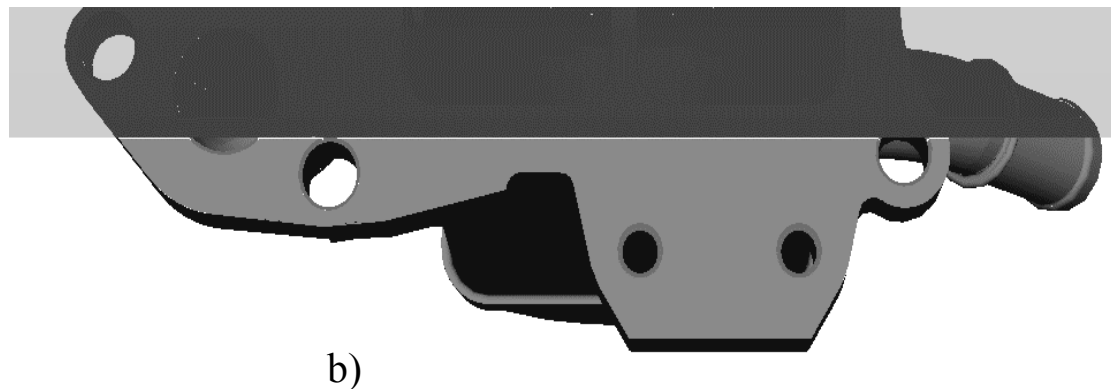

b)

Figure 2: a) Laser stripes on the part surface, b) Direct 3D scanning simulation.

Once the whole system is modelled, the output has to be processed to quantify whether the simulation has achieved the following requirements:

- The data cover the intended surfaces completely.

- Collision-free movements can be guaranteed with an additional security range.

- The results have the resolution necessary for verification of the tolerances.

- The scanning time is suitable for the manufacturing process.

The dual camera configuration shown in Fig. 3 [24] is used as an actual example in verification of the surface flatness.
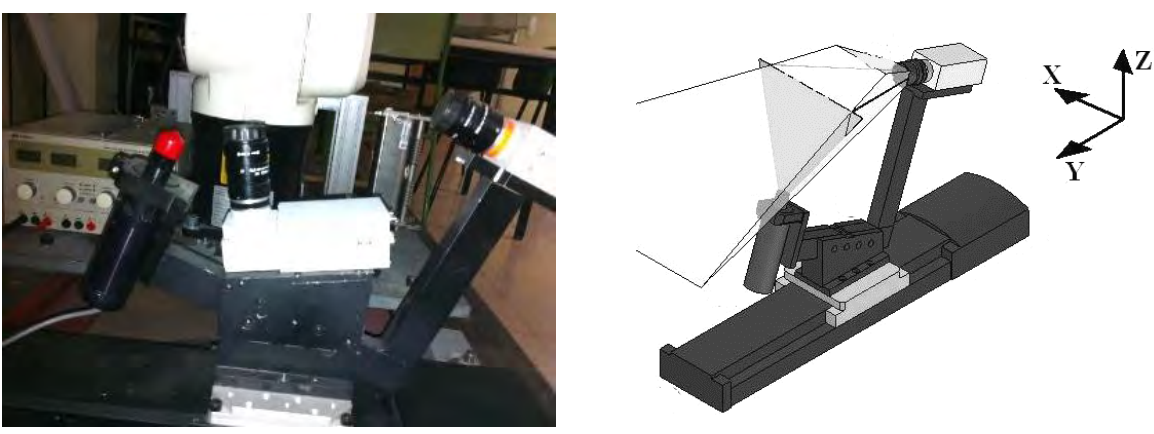

Figure 3: Example of Laser Triangulation Sensor used for dimensional quality assurance in industry.

In addition to the previous requirements verification, thanks to the simulation, our system can also be used to obtain an uncertainty evaluation of the measurement process using numerical methods. In that sense, following the probability distribution propagation calculation recommended by the Guide to the Expression of Uncertainty of Measurement (GUM) [25] and based on the Monte Carlo method, the uncertainty of the system can be estimated. In this case, the uncertainty is not obtained analytically; rather a numerical approach is used to analyse the influence of the input variables, covering their maximum possible range of values, and determines the possible outputs. This process will give the most probable output value and its confidence interval, which will permit the establishment of the related uncertainty.

\section{LST MODEL AND IMPLEMENTATION}

\subsection{Camera}

The most widely applied method for camera modelling is the basic pin-hole camera model, based on the perspective projection principle. As shown in Fig. 4 [7], every 3D point $(X w, Y w, Z w)$ within the field of view has its correspondence in the 2D image plane $(u, v)$. These $u, v$ coordinates are obtained as the projection of the point into the image plane through the optical centre of the camera-optic combination. 


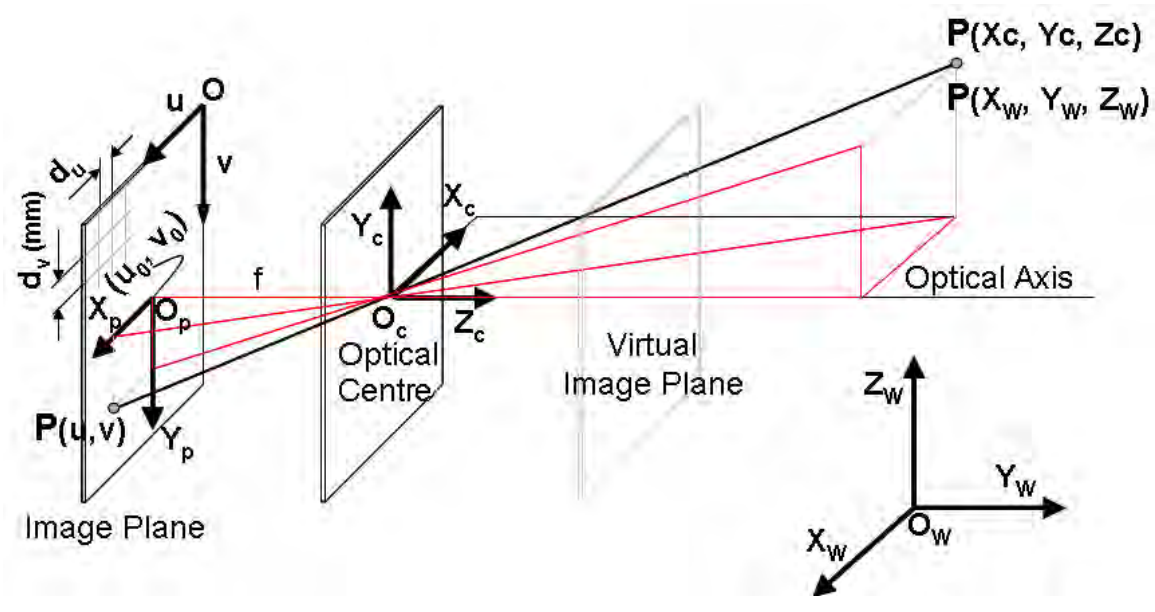

Figure 4: Pin-hole camera model without distortion.

The 3D (real world) to 2D (image) transformation is made from the intrinsic and extrinsic camera parameters [26]. Basic intrinsic parameters refer, first of all, to the characteristics and optics of the camera, involving focal length $f, u_{0}$ and $v_{0}$ coordinates of the principal point in pixels, $k_{i}$ distortion parameters according to the distortion model chosen, and also according to the digital sensor geometry, involving an aspect ratio which determines the length $k_{u}$ and width $k_{v}$ (in $\mathrm{mm}$ ) of the sensor pixels. The extrinsic parameters determine the position and orientation of the camera in the LTS's global coordinate system, expressed in any of the possible formulations of transformation between coordinate systems. The perspective projection principle for camera modelling is shown in (1-2):

$$
\begin{gathered}
{\left[\begin{array}{l}
u \\
v
\end{array}\right]=\left[\begin{array}{ccc}
k_{u} & 0 & u_{0} \\
0 & k_{v} & v_{0}
\end{array}\right] \cdot\left[\begin{array}{c}
X_{p} \\
Y_{p} \\
1
\end{array}\right]} \\
{\left[\begin{array}{l}
X_{p} \\
Y_{p}
\end{array}\right]=\left[\begin{array}{ll}
f & 0 \\
0 & f
\end{array}\right] \cdot\left[\begin{array}{c}
X_{c} \\
Z_{c} \\
\frac{Y_{c}}{Z_{c}}
\end{array}\right]}
\end{gathered}
$$

The 3D coordinates of each point $\left(X_{w}, Y_{w}, Z_{w}\right)$ can be expressed in the camera coordinate system $\left(X_{c}, Y_{c}, Z_{c}\right)$ with (3), where $r_{i j}$ and $t_{x}, t_{y}, t_{z}$ correspond to the rotation and translation terms of a rototranslation matrix respectively. Furthermore, through the perspective transformation matrix (PTM), the image 2D coordinates $(s u, s v)$ of the point are obtained (4). $(u, v)$ correspond to the image coordinates, horizontal and vertical respectively, while $s$ is a scale factor:

$$
\begin{gathered}
{\left[\begin{array}{c}
X_{c} \\
Y_{p} \\
Z_{c}
\end{array}\right]=\left[\begin{array}{llll}
r_{11} & r_{12} & r_{13} & t_{x} \\
r_{21} & r_{22} & r_{23} & t_{y} \\
r_{31} & r_{32} & r_{33} & t_{z}
\end{array}\right] \cdot\left[\begin{array}{c}
X_{w} \\
Y_{w} \\
Z_{w} \\
1
\end{array}\right]} \\
{\left[\begin{array}{c}
s u \\
s v \\
s
\end{array}\right]=P T M \cdot\left[\begin{array}{c}
X_{w} \\
Y_{w} \\
Z_{w} \\
1
\end{array}\right]}
\end{gathered}
$$

According to the obtained equations, the camera field of view can be modelled as a pyramid, as shown in Fig. 5 a. 

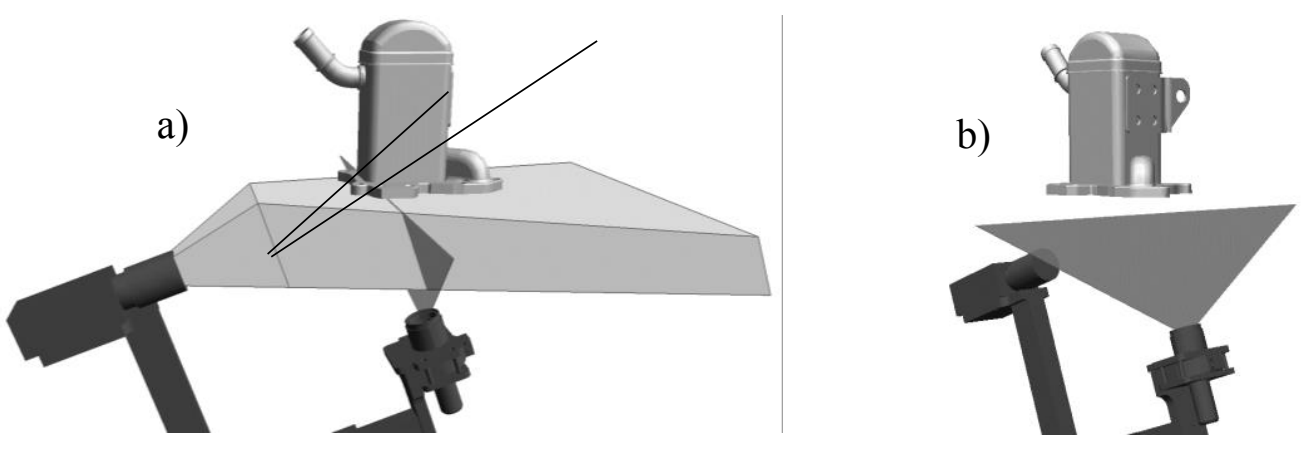

Figure 5: a) Camera field of view modelling [7], b) Triangle for modelling laser plane.

\subsection{Laser}

The main purpose of an LTS is to obtain the 3D coordinates of the projection of the laser on the surface of the part. Therefore, the image points corresponding to the laser have to be unequivocally identified in the image. The laser used in the LTS is modelled by a plane identified by its general equation expressed in the world coordinate system (5). These points in the world reference system match at the same time the camera equations and the laser one. In this way, the part surface, identified by the laser light, is digitalized. Relative movement between the LST and the part is performed until the surface is fully covered:

$$
c A X w+c B Y w+c C Z w+c D=0
$$

As explained in the previous paragraphs, an image point with coordinates $(u, v)$ is modelled by a straight line with two non-parallel plane equations. The laser plane supplies the additional information necessary for the completion of the camera model straight line equation, thus establishing a system of three equations with three variables for each point identified, in order to obtain their 3D world coordinates from their 2D image coordinates $(u, v)$ (Fig. 4). Since the projected stripe originates from a single point, a triangle on that plane is a more accurate modelling than a plane (Fig. 5 b).

\subsection{Parts}

One of the main advantages of the method proposed in this paper is that the CAD of the part is external to the system and therefore there is no limitation regarding the complexity and variety of models. Therefore, the LST system has to be designed considering that not only ideal parts will be digitalized. Instead, instances with discrepancies from the original CAD have to be included. Thus, provided that the dimensions of the part instance are within tolerances, the LST has to be capable of measuring them. Likewise, discrepancies in the positioning due to geometrical variability of instances need to be covered as well. These discrepancies are implemented by scaling the CAD files of each part to the required admissible size. Afterwards, the parts are imported into the simulation environment in "X" file format from any CAD software.

\subsection{Movement}

As mentioned above, another relevant advantage of LTS is that a single capture provides several digitalized points. In a configuration like the one in Fig. 6, the maximum quantity of digitalized points equals the horizontal resolution of the camera. However, to completely measure a surface, additional captures need to be performed. This scanning is achieved with the relative movement between the LTS and the part. Depending on the size and weight of the part, it could be considered more convenient to move the LTS, but the outcome is the same. The movement is modelled by the classical rototranslation matrix applied either to the LST or 
to the part. The rototranslation matrix (6) combines a translation (3 elements) with a rotation (9 elements). Consequently, any possible degree of freedom (linear or angular) is covered:

$$
\left[\begin{array}{c}
X_{w i} \\
Y_{w i} \\
Z_{w i}
\end{array}\right]=\left[\begin{array}{llll}
r_{11} & r_{12} & r_{13} & t_{x} \\
r_{21} & r_{22} & r_{23} & t_{y} \\
r_{31} & r_{32} & r_{33} & t_{z}
\end{array}\right] \cdot\left[\begin{array}{c}
X_{w i-1} \\
Y_{w i-1} \\
Z_{w i-1} \\
1
\end{array}\right]
$$
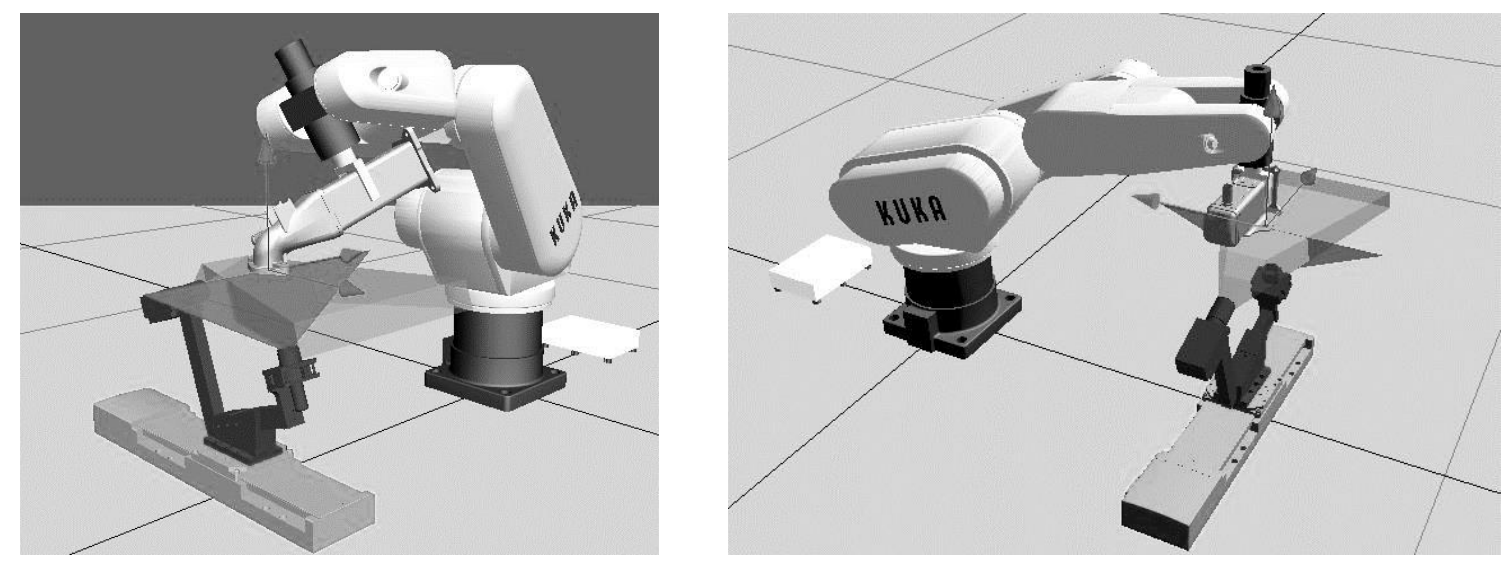

Figure 6: Lineal scanning and robot positioning in Kuka Sim Pro CAM.

After each image frame is captured, the incremental RT matrix has to be applied to the 3D output coordinates in the previous world reference system $\left(X_{w i-1}, Y_{w i-1}, Z_{w i-1}\right)$.

\subsection{Non-ideal effects}

There are many effects that can be considered as a source of uncertainty that may take place in actual LST operation. These effects are usually random, and the simplest approach is to consider their contribution as a probabilistic distribution. If the distribution is Gaussian, each effect is considered as a noise modelled by a random variable with a mean $\mu$ and a standard deviation $\sigma$. Assuming each uncertainty source to be independent allows us to introduce each one in the model separately. The main non-ideal effects that cause uncertainty in the measurements are:

- Laser stripe image misidentification.

- Optical distortion uncertainty.

- Sensor electronic noise.

- Lack of repeatability in positioning of the part.

- Unsynchronized position tracking with the image triggering. The image is not captured at the same time as the position is.

The digitalization requires the identification in the image of the points where the laser plane intersects with the part surfaces. The laser light is not reflected on the surface in a homogeneous way. Then, the image coordinates of the laser are not univocally identified despite the use of elaborate centroid algorithms [27]. Undesired effects of the scan depth and, more importantly, of the projected angle are not considered in the standard calibration procedure. The resulting digitalizing error in the laser-scanned data represents a combination of random error and systematic error [28]. Simulation of the part surface reflection is beyond the scope of this paper. So, a Gaussian noise generator is used to model the laser misidentification. This only affects the $v$ coordinate because of the centroid algorithm used in the identification, resulting in a $2 \mathrm{D}$ vertical coordinate affected by the laser noise $v_{l n}(7)$ :

$$
v_{l n}=v+\text { RandomGaussian }\left(\sigma_{l}, 0\right)
$$


On the other hand, optical distortions are mainly caused by imperfections of cameras and their optics [18], which prevent the camera model being fully correct. The effect of these imperfections in the 2D coordinates $(u, v)$ can be modelled as $(8-10)$ :

$$
\begin{gathered}
u_{d}=\left(1+a_{0} r^{2}+a_{1} r^{4}+a_{2} r^{6}+a_{3} r^{8}+a_{4} r^{10}\right) u+\left(p_{0}+p_{2} r^{2}\right)\left(r^{2}+2 u^{2}\right) \\
+2\left(p_{1}+p_{3} r^{2}\right) u v+s_{0} r^{2}+s_{2} r^{4} \\
v_{d}=\left(1+a_{0} r^{2}+a_{1} r^{4}+a_{2} r^{6}+a_{3} r^{8}+a_{4} r^{10}\right) v+\left(p_{1}+p_{3} r^{2}\right)\left(r^{2}+2 v^{2}\right) \\
+2\left(p_{0}+p_{2} r^{2}\right) u v+s_{1} r^{2}+s_{3} r^{4} \\
r^{2}=\left(u-u_{0}\right)^{2}+\left(v-v_{0}\right)^{2}
\end{gathered}
$$

Where $a_{n}, s_{n}$ and $p_{n}$ represent the $n$ coefficients of radial, prism and tangential distortion respectively. Radial distortions are caused by the paraxial approximation. When light enters into the system with a large angle from the optical axis, the path approximation assumed in the modelling does not represent the reality. Secondly, prism distortions are due to the lack of parallelism between the lenses and the camera sensor. Finally, tangential distortions are attributable to the lack of coaxiality of the lenses composing the optics.

Similarly, electronic noise is known to be an example of white Gaussian noise, so the sensor image noise is considered by a standard deviation and zero mean. In order to properly model this noise, it has to be added on the grey-scale image matrix, conditioning the result of the laser stripe identification process. Since this process is not included in the simulation, the sensor noise becomes part of the previously addressed laser noise modelling.

Finally, lack of repeatability in positioning and position tracking that is unsynchronized with image triggering are exclusively $3 \mathrm{D}$ effects, and so have to be included either in the 3D simulation or in the triangulation calculation (Fig. 1). Both effects are considered as axisindependent random Gaussian error distributions, and these effects can be combined (11), altering the final $3 \mathrm{D}$ coordinates of the points $\left(X^{\prime}{ }_{w i}, Y^{\prime}{ }_{w i}, Z^{\prime}{ }_{w i}\right)$ :

$$
\left[\begin{array}{c}
X^{\prime}{ }_{w i} \\
Y^{\prime}{ }_{w i} \\
Z^{\prime}{ }_{w i}
\end{array}\right]=\left[\begin{array}{c}
X_{w i} \\
Y_{w i} \\
Z_{w i}
\end{array}\right]+\left[\begin{array}{l}
\text { RandomGaussian }\left(\sigma_{x}, \mu_{x}\right) \\
\text { RandomGaussian }\left(\sigma_{y}, \mu_{y}\right) \\
\text { RandomGaussian }\left(\sigma_{z}, \mu_{y}\right)
\end{array}\right]
$$

where $\sigma$ is the standard deviation and $\mu$ is the mean of the Gaussian distribution.

\section{SIMULATION AND EVALUATION}

Using the modelling explained in the previous paragraph, the simulation application has been developed. The implementation has been performed in Visual C++ 2010 with the DirectX 9 SDK from Microsoft. In particular, the direct 3D functions and data framework have been used simultaneously for simulation display and output generation. In addition, a calculator application has been developed with a dialog-based MFC graphic user interface for basic system design.

\subsection{Selection of image acquiring devices}

Regarding image acquisition, the most important design decision is the selection of the camera and its optics. Independently of the optics quality, the main characteristic of an optic is the focal length that, in combination with distance to the target, determines the caption area of the image. A higher focal length provides higher amplification of the image, which reduces the caption area. In addition, a higher focal length usually suffers less optical distortion.

On the other hand, regarding the camera, the type and size of the sensor and the resolution (horizontal and vertical) are the main characteristics. The main categories of image sensors are CCD and CMOS. CCD sensors have lower image noise, which is very important in 
limited illumination conditions, at a higher cost. However, CMOS sensors have the possibility of restricting the capture area to a certain fragment of the sensor, which allows reduction of the frame acquisition time and a consequent increase in the maximum frame rate.

Combining the optic and camera sensor size, the field of view (FOV) of the camera (horizontal and vertical) can be calculated (12):

$$
\propto=2 \arctan \frac{d}{2 f}
$$

where $\alpha$ is the vision angle for a certain dimension, $f$ is the focal length of the optic and $d$ corresponds to the size of the sensor in the same dimension.

Then, the FOV of the camera can be modelled from the sensor size, focal length and distance to the target. Camera sensors are generally not squared, with an aspect ratio $(A R)$ of $5: 4,4: 3$ or 16:9. The diagonal of the rectangle is the dimension used to name the size of the sensor, with its value in a fraction of inches $(1 / 2,1 / 1.7,1 / 1.6,2 / 3,1$, etc.).

The resolution of the sensor is even more important than the size, and it usually follows the same aspect ratio with a certain horizontal resolution (HRes) and a vertical resolution(VRes). Higher resolution increases the density of digitalized surface points as shown in the example of Fig. 7. Using the distance to the target, the FOV and the resolution, the dimensional scale of the caption area can be calculated. Projecting this dimensional scale to the world reference system planes gives the $\mathrm{X}, \mathrm{Y}$ and $\mathrm{Z}$ dimensional resolution.
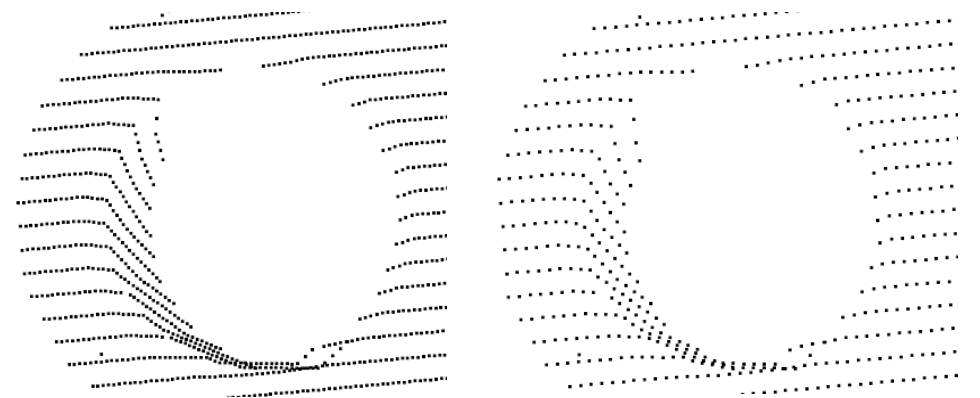

Figure 7: Comparison of horizontal resolution, 1280 vs 640 pixels in LST digitalization simulation.

The combination of the camera FOV with the resolution, tilt and distance to the target permits the calculation of the size of each image pixel in the real world. Even using sub-pixel techniques [27], the 3D size of the pixel determines the resolution of the digitalizing system. Fig. 8 shows a representation of the same camera with different optics. In both cases, the camera is a $1280 \times 1024$ with a $2 / 3$ " sensor.
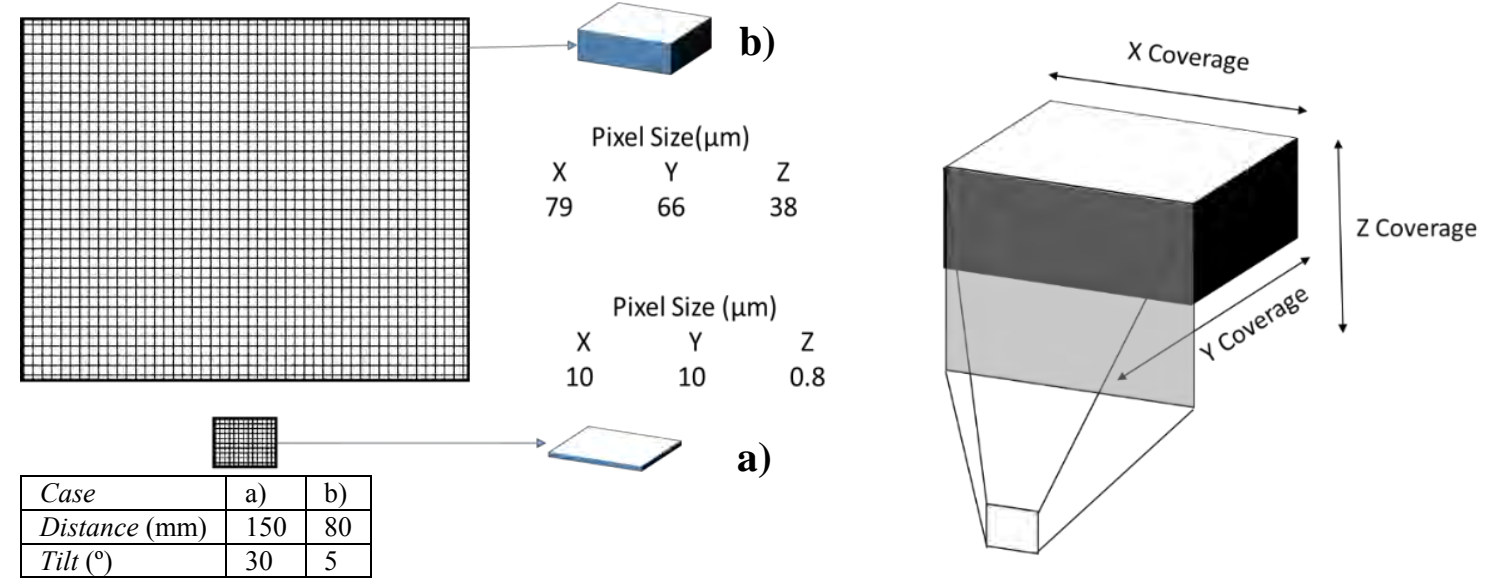

Figure 8: FOV and pixel resolution. 
For the same sensor resolution and size, the coverage area changes, so it also changes the size of each pixel. Smaller pixels give higher measurement resolution.

\subsection{Basic design}

As mentioned above, the devices that compose the system have to be chosen. This process includes the selection of, at least, the camera, optics, movement equipment, laser and the disposition of all of them. In order to verify this initial requirement, we have developed, under Visual Studio 2010, a design assistant application. This application takes care of the basic calculations in the device selection and its results will be used in the actual scanning process simulation. The basic spatial disposition parameters are presented in Fig. 9.

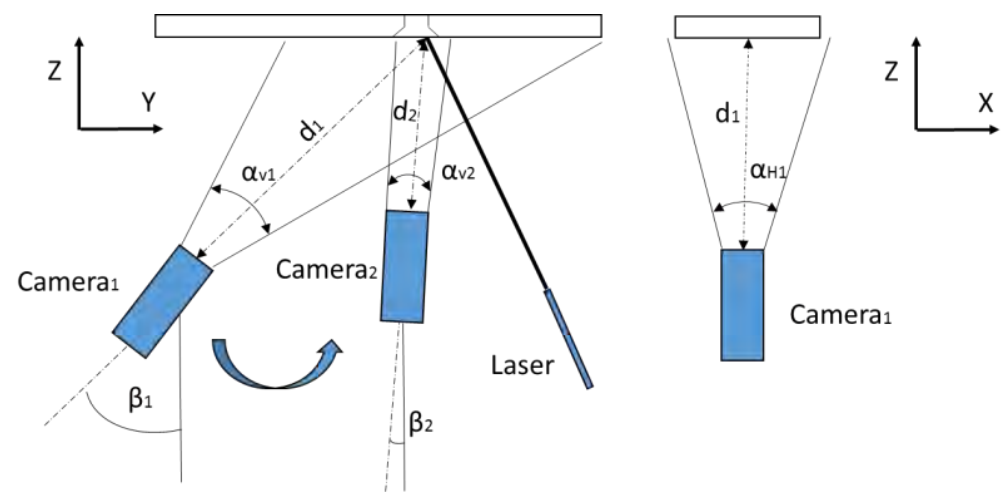

Figure 9: Basic design parameters for a dual camera LST.

As explained, the optic and camera determine the horizontal $\left(\alpha_{H}\right)$ and vertical $\left(\alpha_{v}\right)$ angle of view. Thus, the main parameters that need to be configured are the distance $(d)$ to the target and tilt $(\beta)$ of the camera. Since the scanning movement follows the XY plane, the critical coverage is in the orthogonal direction to the movement axis. In the case of Fig. 9, the system is designed to fully cover the $\mathrm{X}$ dimension of the area of interest (13). In some cases, this area of interest covers the complete part surface, while in others it is restricted to specific features of the part.

$$
d=\frac{F O V}{2 \tan \left(\frac{\alpha_{H}}{2}\right)}
$$

Alternatively, instead of calculating the distance to cover the $\mathrm{X}$ dimension it can be done in the opposite way, obtaining the coverage and pixel size at a certain distance based on the camera parameters and tilt angle. The tilt of the camera determines how the vertical resolution is split between the $\mathrm{Y}$ and $\mathrm{Z}$ axis through its cosine (Fig. 10). With the help of (14-20) the coverage and the pixel size in every dimension can be calculated.

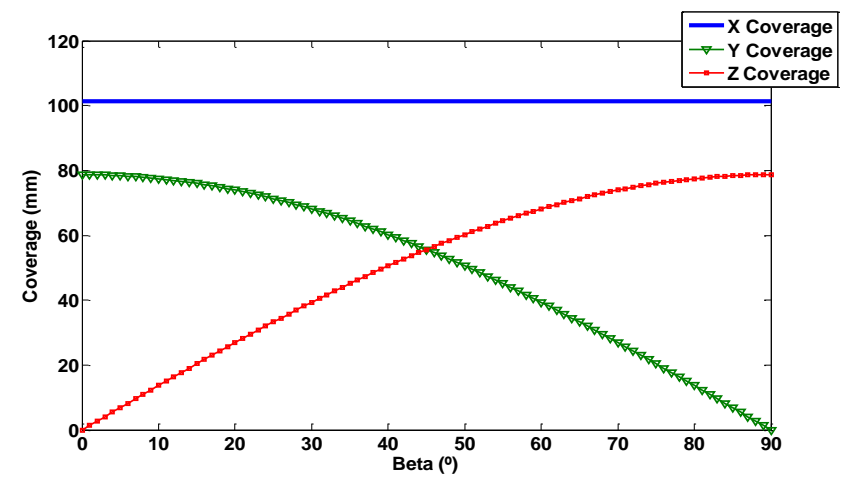

Figure 10: Coverage as a function of $\beta$ for $d=150 \mathrm{~m}, 2 / 3$ " sensor and $f=50 \mathrm{~mm}$. 
The coverage in the $\mathrm{X}$ dimension $\left(\operatorname{COV}_{X}\right)$ can then be calculated and consequently the pixel size $\left(P S_{X}\right)$ in that dimension can also be calculated:

$$
\begin{gathered}
C O V_{X}=2 d \cdot \tan \left(\frac{\alpha_{H}}{2}\right) \\
P S_{X}=\frac{\operatorname{COV}_{X}}{2 H \operatorname{Res}}
\end{gathered}
$$

The coverage in the vertical dimension of the camera $\left(\mathrm{COV}_{V}\right)$ has to be projected on the part to have actual coverage of the part in the $\mathrm{Y}\left(\mathrm{COV}_{Y}\right)$ and $\mathrm{Z}\left(\mathrm{COV}_{Z}\right)$ axes (16-20):

$$
\begin{gathered}
\operatorname{COV}_{V}=2 d \cdot \tan \left(\frac{\alpha_{V}}{2}\right) \\
\operatorname{COV}_{Y}=\operatorname{COV}_{V} \cdot \cos \beta \\
P S_{Y}=\frac{\operatorname{COV}_{Y}}{2 V R e s} \\
\operatorname{COV}_{Z}=\operatorname{COV}_{V} \cdot \sin \beta \\
P S_{Z}=\frac{\operatorname{COV}_{Z}}{2 V \operatorname{Res}}
\end{gathered}
$$

In LTS applications that aim to verify surface flatness, the $\mathrm{Z}$ resolution is a requirement, so the tilt can be calculated from the rest of the design parameters (21):

$$
\beta=\sin ^{-1}\left(\frac{P S_{Z} \cdot V R e s}{2 d \tan \frac{\alpha_{V}}{2}}\right)
$$

\subsection{Scanning}

First of all, the scanning simulation has to address collision detection and data acquisition simulation.

In collision detection, using any computer-assisted manufacturing (CAM) software available in the market, the devices along with the parts and the auxiliary elements (grippers, clamps or frames) can be simulated to detect potential collision. This simulation serves to prevent damage to any components during the measurement operation.

Alternatively, the acquisition simulation retrieves the images of the laser on the surface of the part and, if needed, the 3D coordinates of the surface points signalled by the laser line. This simulation does cover the collision of virtual components such as image pyramids and laser triangles with the part, known as occlusion in the literature.
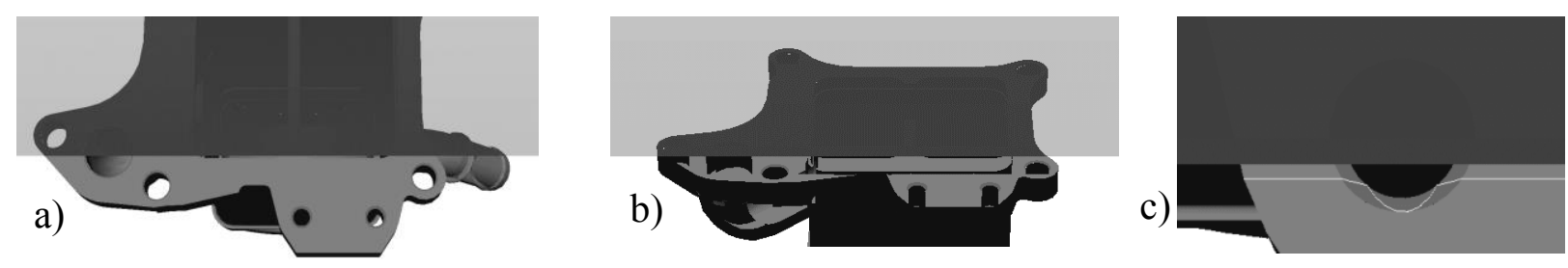

Figure 11: Scanning process simulation of various LST designs.

Fig. 11 shows three simulations with different design parameters. Fig. $11 \mathrm{c}$ includes in white the laser intersection with the part surface that generates the $2 \mathrm{D}$ and $3 \mathrm{D}$ output of the simulation.

The parameters and elements that can be varied in the scanning simulation are:

- Devices and parts,

- Disposition of the elements (part, laser camera), 
- Trajectory (speed, position, orientation),

- Error sources.

\subsection{Frame rate}

This feature is inversely related to scanning speed, with some consideration. Increasing frame rate implies a reduced exposure time of the sensor, which reduces the luminosity of the image and decreases any unwanted motion blur effect. However, reducing the luminosity amplifies sensor electronic noise. Besides, a faster frame rate could also cause additional desynchronization error if the positioning tracking system is not fast enough. Fig. 12 shows two frame rate output examples.
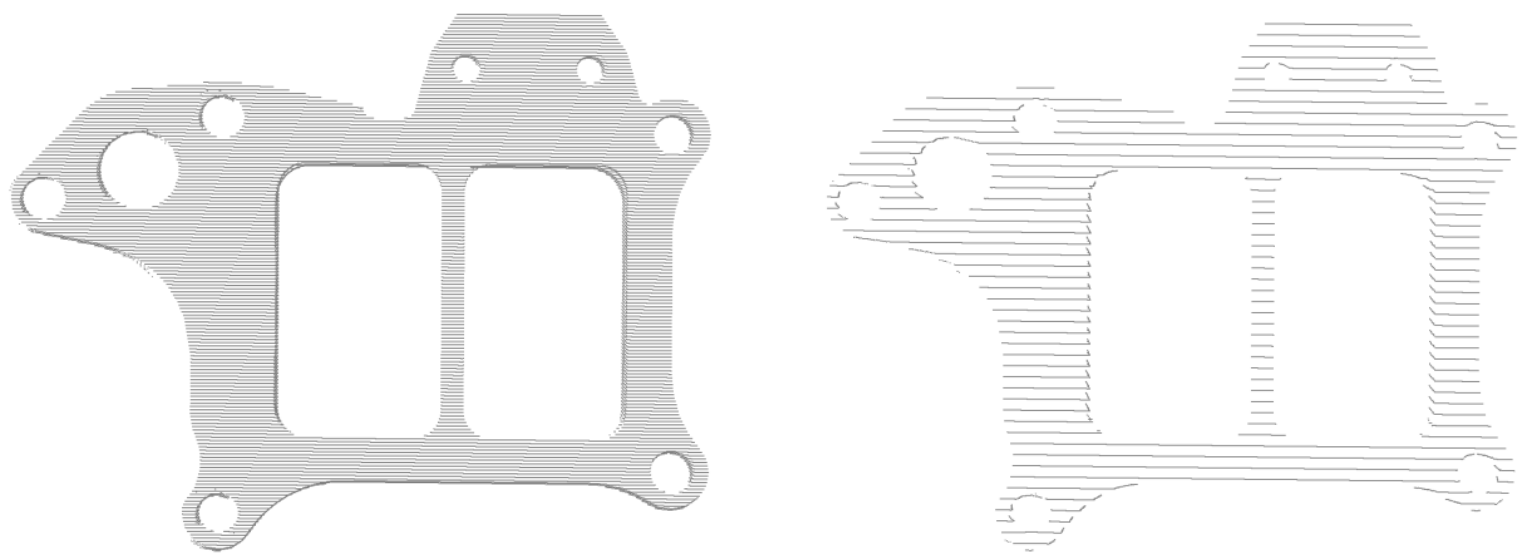

Figure 12: Surface digitalization at $100 \mathrm{fps}$ vs $30 \mathrm{fps}$.

Taking as an example the measurement of a bore-hole centre in [8], it is easy to understand that a denser sampling strategy leads to more accurate results. Therefore, seeking an appropriate trade-off between inspection time and resolution, a custom frame rate strategy can be put in place (Fig. 13). As we have previously stated, the frame rate and scanning speed are in some respects equivalents, but in this particular case, instant speed movement variation requires infinite acceleration, which is not possible. This acceleration would make some image captures a waste and therefore a variable frame rate is more suitable.

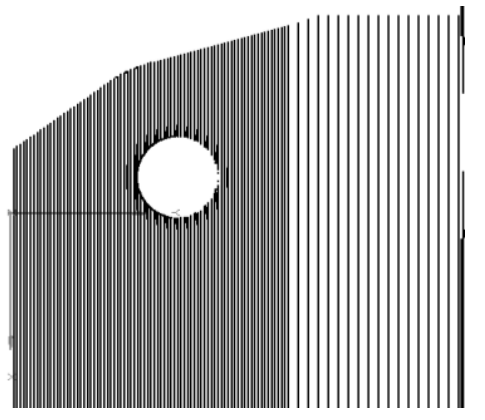

Figure 13: Variable frame rate surface digitalization for measurement of a bore-hole centre.

\subsection{Region of interest (ROI)}

A particularity of CMOS sensors, not shared by CCD sensors, is that the image area to be captured in each frame can be reduced. This feature increases the maximum frame rate, so reducing inspection time at the cost of losing a percentage of the image area. The image area, known as the ROI, can be customized in real time. This is a very interesting feature in LTS, since the effective vertical resolution is always proportional to the coordinate range of the surface in the normal direction of the motion plane. Likewise, optical distortion values 
increase as the pixels move away from the centre of the image because typically the distortion is of a mainly radial nature.

\subsection{Data output of the simulation}

The scanning generates two types of output: the $2 \mathrm{D}(u, v)$ coordinates of the laser on the surface of the part, and the $3 \mathrm{D}$ intersection of the laser plane with the part surface. The $2 \mathrm{D}$ output can include distortion and overlapped noise. Image noise includes optic imperfection due to non-homogeneous surface reflectivity, specular and sensor electronic noise and saturation. The 3D output relies on ideal conditions where the laser and image acquisition are perfect. Nevertheless, unsynchronized effects can be included. As explained in the movement modelling section, between each frame an incremental rototranslation is performed, so any lack of synchronization between position tracking and image capture entails a source of uncertainty.

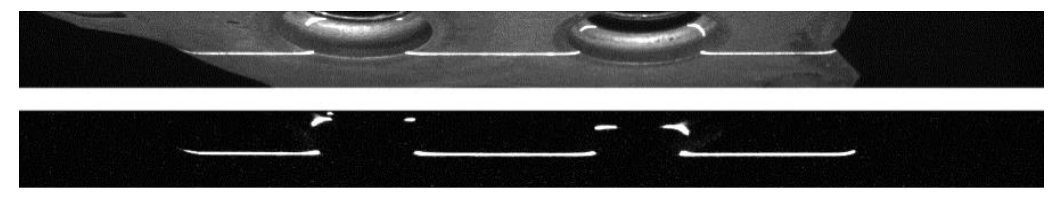

Figure 14: Image acquisition of the LST.

Summarizing the outcomes of the simulation, it provides a very realistic re-creation of an actual image acquisition process (Fig. 14), as well as 2D laser-identified coordinates and 3D triangulated coordinates to assist in the various levels of the LTS scanning tolerance evaluation process.

\section{CONCLUSIONS}

Prototyping is one of the most important steps in the design phase of a product. It permits reevaluation prior to final production while reducing the final cost of the product. The use of $\mathrm{CAM}$ in these processes reduces the time required and improves the general efficiency of the process. The methodology presented in this paper can be considered as equivalent to CAM in LST design. In precision metrology, just the adjustment of the devices takes a huge amount of time from highly trained personnel. The market options of the potential devices and the extent of the parameters to be considered make actual LST prototyping extremely complex. In practice, designers have to reduce the possibilities to a narrow range of available devices and a limited set of frameworks. However, the days required for each spatial disposition become minutes with the method proposed in this paper, even leaving out of the equation the lead delivery time of the hardware components, which quite often come from abroad. The design of the measurement system/strategy can be improved by simulating several devices (camera, optics, guides...), different operating parameters (ROI, speed, frame rate...), and multiple trajectories (distance, orientation, acceleration...), to reduce the process time, improve the data quality and ensure the effectiveness of the process with the most economic approach. As stated, the output of the system, outlined in 4.6, can be used in numeric estimations of the uncertainty of the measurement process.

On the other hand, as mentioned, image acquisition is just one of the steps in visual inspection. The problem is that every single step forward depends on the result of the image acquisition step. This is a bottleneck that impedes simultaneous progress in the subsequent processes of image processing, feature extraction and decision-making. By means of the proposed methods, it is possible to generate source data, allowing the whole implementation to progress at the same time. The feature analysis task receives a cloud of points to work into 
the design of the algorithm for feature extraction and analysis. It has to be taken into consideration that usually the teams engaged in each phase are specific, which can lead to overlapping of interdependent tasks. Furthermore, the simulator allows continuous interference-free process improvement, not involving any extra load in the production chain.

Our aim is that this paper should help quality control designers, enlarging their design possibilities and helping them to tailor the design parameters to the measurement requirements. Also, this paper will enable the quality assurance process to become a key point in the design of parts, where verification concerns can be included alongside functional concerns.

As a principal line of future work, there remains the texturization of parts and modelling of the laser as a real light source to simulate reflection, taking advantage of the rendering capabilities of modern graphical engines.

\section{REFERENCES}

[1] Tirkel, I.; Rabinowitz, G. (2014). Modeling cost benefit analysis of inspection in a production line, International Journal of Production Economics, Vol. 147, 38-45, doi:10.1016/ j.ijpe.2013.05.012

[2] Malamas, E. N.; Petrakis, E. G. M.; Zervakis, M.; Petit, L.; Legat, J.-D. (2003). A survey on industrial vision systems, applications and tools, Image and Vision Computing, Vol. 21, No. 2, 171-188, doi:10.1016/S0262-8856(02)00152-X

[3] Molleda, J.; Usamentiaga, R.; García, D. F.; Bulnes, F. G.; Espina, A.; Dieye, B.; Smith, L. N. (2013). An improved 3D imaging system for dimensional quality inspection of rolled products in the metal industry, Computers in Industry, Vol. 64, No. 9, 1186-1200, doi:10.1016/ j.compind.2013.05.002

[4] Lee, K. H.; Park, H.-P. (2000). Automated inspection planning of free-form shape parts by laser scanning, Robotics and Computer-Integrated Manufacturing, Vol. 16, No. 4, 201-210, doi:10.1016/S0736-5845(99)00060-5

[5] Weckenmann, A.; Knauer, M.; Kunzmann, H. (1998). The influence of measurement strategy on the uncertainty of CMM-measurements, CIRP Annals - Manufacturing Technology, Vol. 47, No. 1, 451-454, doi:10.1016/S0007-8506(07)62872-8

[6] Novak-Marcincin, J.; Torok, J.; Novakova-Marcincinova, L.; Barna, J.; Janak, M. (2014). Use of alternative scanning devices for creation of 3D models of machine parts, Technical Gazette, Vol. 21, No. 1, 177-181

[7] Santolaria, J.; Pastor, J. J.; Brosed, F. J.; Aguilar, J. J. (2009). A one-step intrinsic and extrinsic calibration method for laser line scanner operation in coordinate measuring machines, Measurement Science and Technology, Vol. 20, No. 4, Paper 045107, doi:10.1088/0957$\underline{0233 / 20 / 4 / 045107}$

[8] Brosed, F. J.; Santolaria, J.; Aguilar, J. J.; Guillomía, D. (2012). Laser triangulation sensor and six axes anthropomorphic robot manipulator modelling for the measurement of complex geometry products, Robotics and Computer-Integrated Manufacturing, Vol. 28, No. 6, 660-671, doi:10.1016/j.rcim.2012.04.002

[9] Son, S.; Park, H.; Lee, K. H. (2002). Automated laser scanning system for reverse engineering and inspection, International Journal of Machine Tools and Manufacture, Vol. 42, No. 8, 889897, doi:10.1016/S0890-6955(02)00030-5

[10] Zussman, E.; Schuler, H.; Seliger, G. (1994). Analysis of the geometrical features detectability constraints for laser-scanner sensor planning, The International Journal of Advanced Manufacturing Technology, Vol. 9, No. 1, 56-64, doi:10.1007/BF01792868

[11] Novak, B.; Babnik, A.; Možina, J.; Jezeršek, M. (2014). Three-dimensional foot scanning system with a rotational laser-based measuring head, Strojniski vestnik - Journal of Mechanical Engineering, Vol. 60, No. 11, 685-693, doi:10.5545/sv-jme.2014.1950

[12] Kout, A.; Müller, H. (2014). Tool-adaptive offset paths on triangular mesh workpiece surfaces, Computer-Aided Design, Vol. 50, 61-73, doi:10.1016/j.cad.2014.01.009 
[13] López de Lacalle, L. N.; Rodríguez, A.; Lamikiz, A.; Celaya, A.; Alberdi, R. (2011). Five-axis machining and burnishing of complex parts for the improvement of surface roughness, Materials and Manufacturing Processes, Vol. 26, No. 8, 997-1003, doi:10.1080/10426914.2010.529589

[14] Mavrinac, A.; Chen, X.; Denzinger, P.; Sirizzotti, M. (2010). Calibration of dual laser-based range cameras for reduced occlusion in 3D imaging, 2010 IEEE/ASME International Conference on Advanced Intelligent Mechatronics, 79-83, doi:10.1109/AIM.2010.5695813

[15] Xi, F.; Shu, C. (1999). CAD-based path planning for 3-D line laser scanning, Computer-Aided Design, Vol. 31, No. 7, 473-479, doi:10.1016/S0010-4485(99)00044-5

[16] Lee, G.; Mou, J.; Shen, Y. (1997). Sampling strategy design for dimensional measurement of geometric features using coordinate measuring machine, International Journal of Machine Tools and Manufacture, Vol. 37, No. 7, 917-934, doi:10.1016/S0890-6955(96)00096-X

[17] Smith, K. B.; Zheng, Y. F. (1998). Accuracy analysis of point laser triangulation probes using simulation, Journal of Manufacturing Science and Engineering, Vol. 120, No. 4, 736-745, doi: $10.1115 / 1.2830214$

[18] Dufour, J.-E.; Hild, F.; Roux, S. (2014). Integrated digital image correlation for the evaluation and correction of optical distortions, Optics and Lasers in Engineering, Vol. 56, 121-133, doi:10.1016/j.optlaseng.2013.12.015

[19] Luo, H.; Xu, J.; Hoa Binh, N.; Liu, S.; Zhang, C.; Chen, K. (2014). A simple calibration procedure for structured light system, Optics and Lasers in Engineering, Vol. 57, 6-12, doi:10.1016/j.optlaseng.2014.01.010

[20] Samper, D.; Santolaria, J.; Brosed, F. J.; Majarena, A. C.; Aguilar, J. J. (2013). Analysis of Tsai calibration method using two- and three-dimensional calibration objects, Machine Vision and Applications, Vol. 24, No. 1, 117-131, doi:10.1007/s00138-011-0398-9

[21] Boehler, W.; Bordas-Vicent, M.; Marbs, A. (2003). Investigating laser scanner accuracy, Proceedings of the XIX $X^{\text {th }}$ CIPA Symposium, 696-701, Antalya

[22] Moroni, G.; Petrò, S. (2014). Optimal inspection strategy planning for geometric tolerance verification, Precision Engineering, Vol. 38, No. 1, 71-81, doi:10.1016/j.precisioneng. 2013.07.006

[23] Colosimo, B. M.; Moroni, G.; Petrò, S. (2010). A tolerance interval based criterion for optimizing discrete point sampling strategies, Precision Engineering, Vol. 34, No. 4, 745-754, doi:10.1016/j.precisioneng.2010.04.004

[24] Brosed, F. J.; Aguilar, J. J.; Guillomía, D.; Santolaria, J. (2011). 3D geometrical inspection of complex geometry parts using a novel laser triangulation sensor and a robot, Sensors, Vol. 11, No. 1, 90-110, doi: $10.3390 / \mathrm{s} 110100090$

[25] ISO (2004). The Guide to the Expression of Uncertainty in Measurement (GUM), International Organization for Standardization

[26] Samper, D.; Santolaria, J.; Majarena, A. C.; Aguilar, J. J. (2010). Comprehensive simulation software for teaching camera calibration by a constructivist methodology, Measurement, Vol. 43, No. 5, 618-630, doi:10.1016/j.measurement.2010.01.009

[27] Welch, S. S. (1993). Effects of window size and shape on accuracy of subpixel centroid estimation of target images, NASA Technical Paper 3331, Langley Research Center, Hampton

[28] Feng, H.-Y.; Liu, Y.; Xi, F. (2001). Analysis of digitizing errors of a laser scanning system, Precision Engineering, Vol. 25, No. 3, 185-191, doi:10.1016/S0141-6359(00)00071-4 